
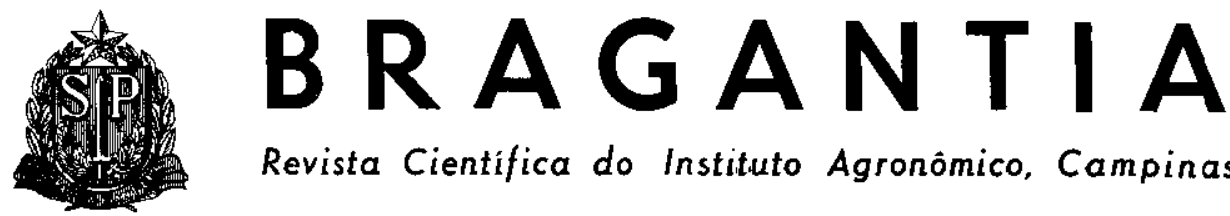

Revista Cientifica do Instituto Agronotmico, Campinas

Vol. 41

Campinas, fevereiro de 1982

Artigo n. ${ }^{\circ} 3$

\title{
EFEITO DA ADUBAÇÃO NPK NA CULTURA DA CROTALÁRIA ( $\left.{ }^{1}\right)$
}

antonio Lulz de Barros Salgado (2), Anf́sto Azzini, Seção de Plantas Fibrosas; Chli Teixeira Feitosa ( $\left.{ }^{2}\right)$, Seção de Fertilidade do Solo; Armando Petingili, E. E. de Tatui, e GUDDo DE SoRDr, E. E. de Ribeirão Preto, Instituto Agronômico

\section{RESUMO}

No presente trabalho são apresentados os resultados obtidos em oito experimentos em que se procurou determinar os efeitos de nitrogênio, fósforo e potássio, na produção de massa verde e sementes de Crotalaria juncea L. No estudo dos três principats elementos, empregou-se um delineamento fatorial $3^{3}$ com duas repetições. Foram estudadas as dosagens de 0,30 e 60 de $\mathrm{N} ; 0,60$ e 120 de $\mathrm{P}_{2} \mathrm{O}_{5}$ e 0,30 e 60 de $\mathrm{K}_{2} \mathrm{O}$, em quilograma/hectare, empregados sob a forma de salitre-do-chile, superfosfato simples e cloreto de potássio respectivamente. Os experimentos foram realizados nos anos agrícolas 1967/68 e 1968/69, nas Estações Experimentais de Campinas, Tietê, Tatú e Ribeirão Preto e, segundo a Comissăo de Solos do Ministério da Agricultura, respectivamente, em solo: Latassolo roxo, série Chapadão; Podzólico vermelho-amarelo variação Piracicaba; Latossolo roxo e Latossolo roxo. Os resultados mostram que, na maioria dos experimentas, o emprego do fósforo aumentou significativamente a produçăo de massa verde $\mathrm{e}$ sementes. $O$ nitrogénio apresentou efeito significativo em alguns deles, ao passo que o efeito do potássio foi praticamente nulo, só apresentando significância quando em presença do fósforo ou do fósforo e nitrogênio juntos.

\section{INTRODUÇÃO}

A partir do momento em que a Crotalaria juncea L. se expandiu, tornando-se cultura econômica no Estado de São Paulo como produtora de fibras para a indústria de celulose e papel, apareceu o problema: qual a melhor adubação a utilizar?
Essa planta começou a ser introduzida com a finalidade de produzir fibras, a partir de 1960; em poucos anos expandiu-se, sendo que em 1968 já era cultivada com esta finalidade em todo o Estado de São Paulo, exceto na região Sul e no Vale do Ribeira.

(1) Trabalho apresentado na XIV Reuniāo Brasileira de Fertilidade do Solo, Cuiabá (MT), 14-19 de julho de 1980 . Recebido para publicaçăo a 9 de maio de 1980.

(2) Com bolsa de suplementação do CNPCT-CNPq. 
Atualmente, São Paulo é o único produtor no Brasil, com o total de 11.000 toneladas de fibras produzidas em mais de 5.000 hectares cultivados, de acordo com SALGADO (4).

A adubação empregada até agora tem sido empírica, não só no que diz respeito à qualidade, como também à quantidade dos fertilizantes. De modo geral, têm-se empregado somente adubações fosfatadas, seja em forma de superfosfato, seja de farinha de ossos e hiperfosfato.

Dada sua crescente importância econômica, é de conveniência a utilização de fórmulas de adubação bem balanceadas que, ao lado de variedades selecionadas e resistentes e práticas agrícolas apropriadas, venham dar maior produção.

O problema đa adubação da crotalária requer um programa extenso de experimentação de pesquisa: este é o primeiro de uma série de trabalhos, nos quais procuramos obter informações para melhor conduzir essa prática agrícola.

A literatura existente é praticamente omissa quanto aos problemas de adubação em C. juncea L., nada existindo a respeito de qualquer tipo de experiência com adubação.

Com o objetivo de estudar a influência dos elementos nitrogênio, fósforo e potássio, na cultura da $\mathbf{C}$. juncea L., instalaram-se em quatro localidades e dois anos agrícolas, os oito experimentos seguintes.

\section{MATERIAL E METODOS}

Os experimentos foram realizados nos anos agrícolas 1967/68 e $1968 / 69$, em diversas regiōes paulistas, nas Estações Experimentais de Campinas, Tietê, Tatuí e Ribeirão Preto, pertencentes ao Instituto Agronômico, em solos classificados respectivamente como: Latossolo roxo, série Chapadão; Podzólico vermelho- amarelo variação Piracicaba; Latossolo roxo e Latossolo roxo (Terra roxa estruturada) (1).

Amostras de terra, retiradas da camada superficial $(0-20 \mathrm{~cm})$, apresentaram as características químicas indicadas no quadro 1 .

Os experimentos foram instalados na época ótima de plantio, outu-

QUADRO 1 - Características químicas das amostras de terra retiradas da camada superficial dos locais dos experimentos

\begin{tabular}{|c|c|c|c|c|c|c|c|}
\hline \multirow{2}{*}{ Ano } & \multirow{2}{*}{ Local } & \multirow{2}{*}{ pH } & \multirow{2}{*}{$\mathbf{C} \%$} & \multicolumn{4}{|c|}{ e. $\mathrm{mg} / 100 \mathrm{ml}$ de T.F.S.A. } \\
\hline & & & & $\mathrm{PO}_{4}^{3}-$ & $\mathbf{K}+$ & $\mathrm{Ca}^{2+}+\mathbf{M g}^{2+}$ & $\mathrm{Al}^{2}+$ \\
\hline \multirow[t]{4}{*}{$67 / 68$} & Campinas & 5,8 & 2,08 & 0,03 & 0,13 & 4,70 & - \\
\hline & Tietê & 5,7 & 1,32 & 0,20 & 0,36 & 4,10 & - \\
\hline & Tatuí & 4,5 & 2,00 & 0,08 & 0,65 & 3,30 & 1,40 \\
\hline & Ribeirão Preto & 5,9 & 2,00 & 0,09 & 0,13 & 4,80 & - \\
\hline \multirow[t]{4}{*}{$68 / 69$} & Campinas & 6,4 & 1,90 & 0,07 & 0,37 & 4,60 & - \\
\hline & Tiete & $\mathbf{5 , 2}$ & 1,20 & 0,09 & 0,41 & 4,70 & - \\
\hline & Tatuí & 5,2 & 1,40 & 0,24 & 0,60 & 4,70 & - \\
\hline & Ribeirāo Preto & 5,7 & 2,01 & 0,07 & 0,18 & 4,60 & - \\
\hline
\end{tabular}


bro/novembro, segundo LOVADINI et alii (2), para perfeita e uniforme germinação das sementes.

Empregou-se a variedade "Comum", que, de longa data, vem sendo cultivada como adubo verde e produtora de fibras, e que MEDINA et alii (3) indicam ser a mais produtiva para São Paulo.

O delineamento experimental usado foi o fatorial $3^{3}$ (NPK), com duas repetiçōes.

Os canteiros apresentavam dez linhas com $5 \mathrm{~m}$ de comprimento, espaçadas de $20 \mathrm{~cm}$, sendo oito úteis $\mathrm{e}$ duas marginais.

A densidade de semeação foi de $9 \mathrm{~g} /$ linha, distribuídos uniformemente no interior dos sulcos, aproximadamente 45 sementes por metro linear.

Os níveis dos elementos fertilizantes empregados, em quilograma/ hectare, foram de 0,30 e 60 de $\mathrm{N}$; 0,60 e 120 de $\mathrm{P}_{2} \mathrm{O}_{5}$ e 0,30 e 60 de $\mathrm{K}_{2} \mathrm{O}$.

O nitrogênio, fósforo e potássio foram empregados em forma de salitre-do-chile, superfosfato simples e cloreto de potássio respectivamente.

Os fertilizantes foram misturados e espalhados uniformemente sobre os respectivos canteiros e incorporados ao solo com rastelo antes do plantio.

\section{RESULTADOS E DISCUSSÃO}

A germinação das sementes de todos os experimentos instalados nas quatro localidades e em dois anos agrícolas foi sempre boa, apresentando stand inicial sem diferenciação entre tratamentos.
Para a avaliação da eficiência dos diversos tratamentos, foram tomadas as produções, em quilograma por hectare, de massa verde e sementes.

A análise da variância para o teste $\mathbf{F}$ dos experimentos realizados será apresentada individualmente, experimento por experimento, para cada local e ano agrícola.

\subsection{Ano agrícola de $1967 / 68$}

Os dados de produção de massa verde e sementes, expressos em quilogramas por hectare, nas diferentes localidades, para o ano agrícola $1967 / 68$, são mostrades respectivamente nos quadros 2 e 3 .

\subsubsection{Centro Experimental de Campinas}

No experimento realizado em Campinas, no ano agrícola 1967/68 para a produção de massa verde, o coeficiente de variação correspondeu a $17,6 \%$.

O nitrogênio não apresentou efeito significativo.

O fósforo aumentou a produção de massa verde, e tanto o componente linear como o quadrático alcançaram significância; o linear ao nível de $1 \%$ e o quadrático ao nível de $5 \%$.

Os aumentos proporcionados por $P_{1}$ e $P_{2}$ elevaram-se respectivamente a $37,3 \%$ e $40,8 \%$.

$O$ efeito do potássio foi nulo para o $K_{1}$ e de $5,8 \%$ para o $K_{2}$, mas não significativo.

As interações duplas não alcançaram significância para 0 teste $F$, assim como a interação tripla. 
Vol. 41 , Art. n. ${ }^{\circ} 3$

QUADRO 2 - Produção de massa verde, calculada em quilograma por hectare, obtida em quatro localidades no ano agrícola $67 / 68$, em experimento de adubaçăo de Crotalária, média de duas repetiçōes

\begin{tabular}{|c|c|c|c|c|}
\hline $\begin{array}{c}\text { Tratamento } \\
\text { (Niveis de } \\
\text { N, P e K) }\end{array}$ & Campinas & Tietê & Tatuí & $\begin{array}{c}\text { Ribeirão } \\
\text { Preto }\end{array}$ \\
\hline $000 \ldots \ldots \ldots \ldots$ & 11.405 & 20.315 & 16.405 & 25.940 \\
\hline $001 \ldots \ldots \ldots$ & 10.705 & 19.610 & 17.035 & 22.500 \\
\hline $002 \ldots \ldots \ldots \ldots$ & 10.315 & 19.920 & 16.875 & 23.830 \\
\hline $010 \ldots \ldots \ldots$ & 14.140 & 20.625 & 29.685 & 24.845 \\
\hline $011 \ldots \ldots \ldots \ldots$ & 17.765 & 21.095 & 16.715 & 23.750 \\
\hline $012 \ldots \ldots \ldots \ldots$ & 17.970 & 22.110 & 16.485 & 25.390 \\
\hline $020 \ldots \ldots \ldots \ldots$ & 15.465 & 21.090 & 16.485 & 19.685 \\
\hline $021 \ldots \ldots \ldots \ldots$ & 17.885 & 19.610 & 18.390 & 26.720 \\
\hline $022 \ldots \ldots \ldots \ldots$ & 15.075 & 19.765 & 19.610 & 25.705 \\
\hline $100 \ldots \ldots \ldots \ldots$ & 10.155 & 21.640 & 7.890 & 23.675 \\
\hline $101 \ldots \ldots \ldots \ldots$ & 9.065 & 25.780 & 17.190 & 24.065 \\
\hline $102 \ldots \ldots \ldots \ldots$ & 13.360 & 22.660 & 27.970 & 24.765 \\
\hline $110 \ldots \ldots \ldots \ldots$ & 16.560 & 21.955 & 10.780 & 22.895 \\
\hline $111 \ldots \ldots \ldots \ldots$ & 13.045 & 25.780 & 22.030 & 25.470 \\
\hline $112 \ldots \ldots \ldots \ldots$ & 17.815 & 22.970 & 17.735 & 23.045 \\
\hline $120 \ldots \ldots \ldots \ldots$ & 16.170 & 25.075 & 22.815 & 26.250 \\
\hline $121 \ldots \ldots \ldots \ldots$ & 14.925 & 22.425 & 16.330 & 21.325 \\
\hline $122 \ldots \ldots \ldots \ldots$ & 13.515 & 25.155 & 16.330 & 27.735 \\
\hline $200 \ldots \ldots \ldots \ldots$ & 11.640 & 19.845 & 10.780 & 23.205 \\
\hline $201 \ldots \ldots \ldots$ & 9.530 & 23.050 & 13.520 & 24.295 \\
\hline $202 \ldots \ldots \ldots \ldots$ & 14.455 & 24.060 & 6.405 & 26.015 \\
\hline $210 \ldots \ldots \ldots \ldots$ & 14.610 & 15.935 & 22.465 & 23.280 \\
\hline $211 \ldots \ldots \ldots \ldots$ & 14.295 & 24.610 & 13.280 & 25.005 \\
\hline $212 \ldots \ldots \ldots \ldots$ & 11.955 & 27.500 & 32.790 & 24.690 \\
\hline $220 \ldots \ldots \ldots \ldots$ & 16.020 & 25.390 & 14.455 & 25.545 \\
\hline $221 \ldots \ldots \ldots \ldots$ & 13.675 & 21.330 & 15.860 & 26.565 \\
\hline $222 \ldots \ldots \ldots \ldots$ & 18.985 & 27.345 & 17.185 & 23.125 \\
\hline \multicolumn{5}{|l|}{ MEDIAS } \\
\hline $\mathbf{N}_{0} \ldots \ldots \ldots \ldots$ & 14.525 & 20.460 & 18.632 & 24.263 \\
\hline $\mathbf{N}, \ldots \ldots \ldots \ldots$ & 13.845 & 23.716 & 17.674 & 24.358 \\
\hline $\mathbf{N}_{2} \ldots \ldots \ldots \ldots$ & 13.907 & 23.229 & 16.304 & 24.636 \\
\hline $\mathbf{P}_{0} \ldots \ldots \ldots \ldots$ & 11.181 & 21.876 & 14.897 & 24.254 \\
\hline$P_{\mathbf{z}} \ldots \ldots \ldots \ldots$ & 15.350 & 22.509 & 20.218 & 24.263 \\
\hline $\mathbf{P}_{\mathbf{2}} \ldots \ldots \ldots \ldots$ & 15.746 & 23.021 & 17.496 & 24.739 \\
\hline $\mathbf{K}_{0} \ldots \ldots \ldots$ & 14.018 & 21.319 & 16.862 & 23.924 \\
\hline $\mathbf{K}_{1} \ldots \ldots \ldots \ldots$ & 13.432 & 22.588 & 16.706 & 24.411 \\
\hline $\mathbf{K}_{2} \ldots \ldots \ldots \ldots$ & 14.827 & 23.498 & 19.043 & 24.922 \\
\hline
\end{tabular}


QUADRO 3 - PToduçâo de sementes, calculada em quilograma por hectare, obtida em quatro localidades no ano agrícola $67 / 68$, em experimento de adubaçăo de Crotalária, média de duas repetiçóes

\begin{tabular}{|c|c|c|c|c|}
\hline $\begin{array}{l}\text { Tratamento } \\
\text { (Niveis de } \\
\text { N, P e K) }\end{array}$ & Campinas & Tietê & Tatuí & $\begin{array}{c}\text { Ribeirāo } \\
\text { Preto }\end{array}$ \\
\hline $\mathbf{0 0 0} \ldots \ldots \ldots \ldots$ & 785 & 1.180 & 415 & 1.425 \\
\hline $001 \ldots \ldots \ldots \ldots$ & 915 & 900 & 285 & 1.310 \\
\hline $\mathbf{0 0 2} \ldots \ldots \ldots \ldots$ & 915 & 940 & 360 & 900 \\
\hline $010 \ldots \ldots \ldots \ldots$ & 1.165 & 625 & 395 & 965 \\
\hline $011 \ldots \ldots \ldots \ldots$ & 1.060 & 1.015 & 400 & 1.030 \\
\hline $012 \ldots \ldots \ldots \ldots$ & 1.395 & 945 & 280 & 1.460 \\
\hline $\mathbf{0 2 0} \ldots \ldots \ldots \ldots$ & 1.110 & 1.015 & 410 & 900 \\
\hline $021 \ldots \ldots \ldots \ldots$ & 1.485 & 900 & 285 & 1.320 \\
\hline $022 \ldots \ldots \ldots \ldots$ & 1.125 & 990 & 540 & 1.235 \\
\hline $100 \ldots \ldots \ldots \ldots$ & 790 & 1.085 & 200 & 1.050 \\
\hline $101 \ldots \ldots \ldots \ldots$ & 830 & 1.460 & 300 & 1.415 \\
\hline $102 \ldots \ldots \ldots \ldots$ & 1.055 & 875 & 675 & 1.195 \\
\hline $110 \ldots \ldots \ldots \ldots$ & 845 & 1.025 & 210 & 1.300 \\
\hline $111 \ldots \ldots \ldots \ldots$ & 1.085 & 1.340 & 505 & 1.260 \\
\hline $112 \ldots \ldots \ldots \ldots$ & 1.245 & 1.040 & 390 & 1.140 \\
\hline $120 \ldots \ldots \ldots \ldots$ & 1.350 & 1.465 & $\mathbf{4 7 0}$ & 1.495 \\
\hline $121 \ldots \ldots \ldots \ldots$ & 1.445 & 1.000 & 330 & 1.300 \\
\hline $122 \ldots \ldots \ldots \ldots$ & 1.250 & 1.300 & 390 & 1.380 \\
\hline $200 \ldots \ldots \ldots \ldots$ & 920 & 735 & 195 & 1.150 \\
\hline $201 \ldots \ldots \ldots \ldots$ & 730 & 1.120 & 465 & 1.375 \\
\hline $202 \ldots \ldots \ldots \ldots$ & 1.165 & 1.030 & 185 & 1.350 \\
\hline $210 \ldots \ldots \ldots \ldots$ & 1.225 & 730 & 460 & 1.065 \\
\hline $211 \ldots \ldots \ldots \ldots$ & 1.080 & 955 & 465 & 1.370 \\
\hline $212 \ldots \ldots \ldots \ldots$ & 1.290 & 1.550 & 600 & 1.180 \\
\hline $220 \ldots \ldots \ldots \ldots$ & 1.095 & 1.100 & 375 & 1.265 \\
\hline $221 \ldots \ldots \ldots \ldots$ & 1.165 & 1.000 & 280 & 1.420 \\
\hline $222 \ldots \ldots \ldots \ldots$ & 1.070 & 1.055 & 335 & 1.050 \\
\hline \multicolumn{5}{|l|}{ MEDIAS } \\
\hline $\mathbf{N}_{1} \ldots \ldots \ldots \ldots$ & 1.106 & 946 & 374 & 1.172 \\
\hline $\mathbf{N}, \ldots \ldots \ldots \ldots$ & 1.099 & 1.177 & 386 & 1.282 \\
\hline $\mathbf{N}_{:}, \ldots \ldots \ldots \ldots$ & 1.082 & 1.031 & 373 & 1.247 \\
\hline $\mathbf{P}_{n} \ldots \ldots \ldots \ldots$ & 901 & 1.036 & 342 & 1.241 \\
\hline $\mathbf{P}, \ldots \ldots \ldots \ldots$ & 1.154 & 1.025 & 412 & 1.197 \\
\hline $\mathbf{P}_{2} \ldots \ldots \ldots \ldots$ & 1.233 & 1.092 & 379 & 1.263 \\
\hline $\mathbf{K}_{,}, \ldots \ldots \ldots \ldots$ & 1.032 & 996 & 348 & 1.179 \\
\hline $\mathbf{K}_{\mathbf{1}} \ldots \ldots \ldots \ldots$ & 1.088 & 1.077 & 368 & 1.311 \\
\hline $\mathbf{K}_{2} \ldots \ldots \ldots \ldots$ & 1.168 & 1.081 & 417 & 1.210 \\
\hline
\end{tabular}


Para a produção de sementes, o coeficiente de variação correspondeu a $16,1 \%$. Houve efeito significativo ao nível de $1 \%$ para os tratamentos.

$\mathrm{O}$ nitrogênio não apresentou efeito significativo na produção.

$O$ fósforo apresentou efeito altamente significativo.

Os aumentos proporcionados pelo $P_{1}$ e $P_{2}$ foram respectivamente de $28,1 \%$ e $36,8 \%$.

O potássio não apresentou significância estatística, mas proporcionou aumentos de $5,4 \%$ e $13,2 \%$ respectivamente para $\mathrm{K}_{1}$ e $\mathrm{K}_{2}$.

A interação dupla PK apresentou significância estatística; as demais interações não apresentaram significância.

\subsubsection{Estação Experimental de Tietê}

$\mathrm{Na}$ experiência realizada em Tietê, no ano agrícola 1967/68, o coeficiente de variação foi $13,3 \%$ para a produção de massa verde.

$O$ nitrogênio aumentou significativamente a produção, o componente linear alcançou significância ao nível de $1 \%$ e o quadrático ao nível de $5 \%$. O aumento proporcionado por $\mathrm{N}_{1}$ foi $15,9 \%$ e o de $\mathrm{N}_{2} 13,5 \%$.

$\mathrm{O}$ efeito do fósforo não foi significativo tanto para o componente linear como quadrático; os aumentos provocados por $\mathbf{P}_{1}$ e $\mathbf{P}_{2}$ foram respectivamente apenas 2,9 e $5,2 \%$.

O efeito linear do potássio foi significativo ao nível de $5 \%$, ao passo que o efeito quadrático não apresentou significância estatística. Os aumentos provocados por $\mathrm{K}_{1}$ e $\mathrm{K}_{2}$ foram respectivamente 5,9 e $10,2 \%$.
As interações duplas e triplas não alcançaram significância.

Para a produção de sementes, o coeficiente de variação foi $19,9 \%$.

$O$ efeito do nitrogênio foi não significativo para o componente linear e significativo estatisticamente ao nível de $1 \%$ para o quadrático. $\mathrm{O}$ aumento proporcionado pelo $\mathrm{N}_{1}$ foi $24,4 \%$, superior ao $\mathrm{N}_{2}$, que foi apenas $9,0 \%$.

O efeito do fósforo não foi significativo.

$\mathrm{O}$ potássio causou aumentos de $8,1 \%$ para o $\mathrm{K}_{1}$ e $8,5 \%$ para o $\mathrm{K}_{2}$, mas sem apresentar efeito significativo.

A interação $\mathbf{P K}$ apresentou efeito altamente significativo, ao contrário das demais interações.

\subsubsection{Estação Experimental de Tatuí}

No experimento realizado durante o ano agrícola $1967 / 68$, na Estação Experimental de Tatuí, para a produção de massa verde, o coeficiente de variação correspondeu a $20,2 \%$.

$O$ efeito do nitrogênio não foi significativo, porém observou-se diminuição na produção, devido ao aumento de doses de nitrogênio.

$O$ efeito linear do fósforo foi significativo ao nível de $5 \%$, ao passo que o do componente quadrático foi altamente significativo. Os aumentos provocados por $\mathbf{P}_{1}$ e $\mathbf{P}_{2}$ foram respectivamente 35,7 e $17,4 \%$.

O potássio não teve efeito significativo tanto para o componente linear como quadrático, mas provocou aumento de $12,9 \%$ na produção quando se utilizou a dose dois de K. 
Nas interações duplas, NP foi altamente significativo, porém houve significância ao nível de $5 \%$ para NK, ao passo que PK não foi significativo. A interação tripla apresentou efeito altamente significativo.

Para a produção de sementes, o coeficiente de variação correspondeu a $26,7 \%$, apresentando efeito altamente significativo para os tratamentos.

O efeito do nitrogênio não foi significativo, tanto para o componente linear como para o quadrático. $\mathrm{O} \mathrm{N}_{1}$ apresentou aumento de produção de $3,2 \%$, ao passo que o efeito do $\mathrm{N}_{2}$ foi nulo.

O fósforo aumentou razoavelmente a produção, mas os seus componentes linear e quadrático não apresentaram efeito significativo. $O$ aumento proporcionado por $\mathrm{P}_{1}$ foi $20,5 \%$ e do $P_{2}, 10,8 \%$.

O efeito linear do potássio foi significativo ao nível de $5 \%$, e os aumentos proporcionados por $\mathbf{K}_{1}$ e $\mathbf{K}_{2}$ foram $5,7 \%$ e $19,8 \%$ respectivamente.

As interações duplas NP e PK foram altamente significativas, enquanto NK alcançou significância a $5 \%$.

O emprego de NPK não apresentou significância estatística.

\subsubsection{Estaçâo Experimental de Ribeirão Preto}

O coeficiente de variação correspondente a $9,1 \%$ foi apresentado pelo experimento instalado no ano agrícola 1967/68 na Estação Experimental de Ribeirão Preto, para a produção de massa verde.
Não houve efeito significativo para os empregos do nitrogênio, fósforo e potássio em seus componentes linear e quadrático, o mesmo ocorrendo com as interações duplas.

Os aumentos proporcionados pelo emprego de N, P e K foram praticamente nulos, correspondendo a $0,4,1,50,0,2,0,2,0$ e $4,2 \%$ respectivamente para $N_{1}, N_{2}, P_{1}, P_{2}$, $\mathrm{K}_{1}$ e $\mathrm{K}_{2}$.

A interação tripla apresentou efeito altamente significativo.

Para a produção de sementes, o coeficiente de variação correspondeu a $16,9 \%$.

Os componentes lineares e quadráticos de $\mathbf{N}, \mathbf{P}$ e $\mathbf{K}$ não apresentaram efeito significativo.

$\mathrm{O}$ emprego de $\mathrm{N}_{1}$ e $\mathrm{N}_{2}$ apresentou aumentos de 9,4 e $6,4 \%$ respectivamente.

$\mathrm{O}$ emprego do $\mathrm{P}_{1}$ foi nulo e o de $P_{2}$, de apenas $1,7 \%$.

A porcentagem da utilização do $K_{1}$ foi 11,2 e de $K_{2}$, apenas $2,6 \%$.

As interações duplas não alcançaram significância, porém NPK apresentou efeito altamente significativo também para a produção de sementes.

\subsection{Ano agrícola de $1968 / 69$}

Nos quadros 4 e 5 são apresentados, respectivamente, os resultados de produção de massa verde e sementes, expressa em quilogramas por hectare, nas localidades de Campinas, Tatuí, Tietê e Ribeirão Preto, para o ano agrícola 1968/69. 
QUADRO 4 - Produçāo de massa verde, calculada em quilograma por hectare, obtida em quatro localidades no ano agrícola de 68/69, em experimentos de adubação em Crotalária, média de duas repetiç̋́es

\begin{tabular}{|c|c|c|c|c|}
\hline $\begin{array}{c}\text { Tratamento } \\
\text { (Niveis de } \\
\mathbf{N}, \text { P e } \mathbf{K} \text { ) }\end{array}$ & Campinas & Tietê & Tatú & $\begin{array}{c}\text { Ribeirão } \\
\text { Preto }\end{array}$ \\
\hline $000 \ldots \ldots \ldots \ldots$ & 22.110 & 19.220 & 8.670 & 18.515 \\
\hline $001 \ldots \ldots \ldots \ldots$ & 21.485 & 12.640 & 13.595 & 22.580 \\
\hline $002 \ldots \ldots \ldots \ldots$ & 20.235 & 11.720 & 9.375 & 26.485 \\
\hline $010 \ldots \ldots \ldots \ldots$ & 29.925 & 14.610 & 12,580 & 20.470 \\
\hline $011 \ldots \ldots \ldots \ldots$ & 18.830 & 16.720 & 14.765 & 21.095 \\
\hline $012 \ldots \ldots \ldots \ldots$ & 21.095 & 18.830 & 10.235 & 22.500 \\
\hline $020 \ldots \ldots \ldots \ldots$ & 24.535 & 15.780 & 13.360 & 22.035 \\
\hline $021 \ldots \ldots \ldots$ & 26.485 & 23.515 & 14.845 & 21.330 \\
\hline $022 \ldots \ldots \ldots \ldots$ & 19.770 & 16.250 & 16.955 & 23.280 \\
\hline $100 \ldots \ldots \ldots \ldots$ & 19.660 & 10.160 & 11.800 & 23.750 \\
\hline $101 \ldots \ldots \ldots \ldots$ & 21.175 & 18.830 & 11.640 & 23.595 \\
\hline $102 \ldots \ldots \ldots \ldots$ & 20.860 & 14.690 & 14.610 & 26.325 \\
\hline $110 \ldots \ldots \ldots \ldots$ & 26.410 & 15.160 & 10.470 & 24.920 \\
\hline $111 \ldots \ldots \ldots \ldots$ & 20.160 & 17.110 & 14.690 & 22.910 \\
\hline $112 \ldots \ldots \ldots \ldots$ & 18.125 & 11.175 & 12.580 & 20.390 \\
\hline $120 \ldots \ldots \ldots \ldots$ & 16.405 & 22.185 & 15.235 & 22.500 \\
\hline $121 \ldots \ldots \ldots \ldots$ & 18.905 & 15.160 & 14.455 & 26.170 \\
\hline $122 \ldots \ldots \ldots \ldots$ & 21.560 & 22.970 & 15.000 & 22.815 \\
\hline $200 \ldots \ldots \ldots \ldots$ & 22.890 & 11.640 & 13.910 & 20.935 \\
\hline $201 \ldots \ldots \ldots \ldots$ & 18.205 & 12.185 & 11.485 & 23.050 \\
\hline $202 \ldots \ldots \ldots \ldots$ & 21.410 & 20.000 & 11.405 & 26.020 \\
\hline $210 \ldots \ldots \ldots \ldots$ & 24.825 & 20.940 & 15.000 & 23.050 \\
\hline $211 \ldots \ldots \ldots \ldots$ & 30.000 & 11.640 & 11.720 & 23.595 \\
\hline $212 \ldots \ldots \ldots \ldots$ & 26.640 & 20.705 & 15.155 & 26.405 \\
\hline $220 \ldots \ldots \ldots \ldots$ & 19.610 & 14.530 & 12.560 & 23.280 \\
\hline $221 \ldots \ldots \ldots \ldots$ & 25.000 & 13.910 & 18.750 & 24.610 \\
\hline $222 \ldots \ldots \ldots \ldots$ & 24.765 & 19.925 & 15.625 & 21.640 \\
\hline \multicolumn{5}{|l|}{ MEDIAS } \\
\hline $\mathbf{N}_{0} \ldots \ldots \ldots \ldots$ & 22.719 & 16.587 & 12.709 & 22.032 \\
\hline $\mathbf{N}_{1} \ldots \ldots \ldots \ldots$ & 20.362 & 16.382 & 13.387 & 23.708 \\
\hline $\mathbf{N}_{2} \ldots \ldots \ldots \ldots$ & 23.705 & 16.164 & 13.957 & 23.621 \\
\hline $\mathbf{P}_{\mathbf{b}} \ldots \ldots \ldots \ldots$ & 20.892 & 14.565 & 11.832 & 23.473 \\
\hline$P_{1} \ldots \ldots \ldots \ldots$ & 24.001 & 16.321 & 13.022 & 22.815 \\
\hline $\mathbf{P}_{\mathbf{a}} \ldots \ldots \ldots \ldots$ & 21.893 & 18.247 & 15.198 & 23.073 \\
\hline $\mathbf{K}_{0} \ldots \ldots \ldots \ldots$ & 22.930 & 16.025 & 12.621 & 22.162 \\
\hline $\mathbf{K}_{1} \ldots \ldots \ldots \ldots$ & 22.249 & 15.746 & 13.994 & 23.215 \\
\hline $\mathbf{K}_{\mathbf{a}} \ldots \ldots \ldots \ldots$ & 21.607 & 17.363 & 13.438 & 23.984 \\
\hline
\end{tabular}


QUADRO 5 - Produçăo de sementes, calculada em quilograma por hectare, obtida em quatro localidades no ano agrícola $68 / 69$, em experiência de adubaçăo em Crotalária, média de duas repetiçóes

\begin{tabular}{|c|c|c|c|c|}
\hline $\begin{array}{c}\text { Tratamento } \\
\text { (Niveis de } \\
\text { N, P e K) }\end{array}$ & Campinas & Tietê & Tatú & $\begin{array}{c}\text { Ribeirão } \\
\text { Preto }\end{array}$ \\
\hline $000 \ldots \ldots \ldots \ldots$ & 1.055 & 375 & 500 & 1.110 \\
\hline $001 \ldots \ldots \ldots \ldots$ & 1.315 & 170 & 710 & 1.335 \\
\hline $002 \ldots \ldots \ldots \ldots$ & 1.365 & 155 & 525 & 1.390 \\
\hline $010 \ldots \ldots \ldots \ldots$ & 1.290 & 430 & 705 & 1.090 \\
\hline $011 \ldots \ldots \ldots \ldots$ & 1.250 & 470 & 710 & 1.180 \\
\hline $012 \ldots \ldots \ldots \ldots$ & 1.325 & 290 & 525 & 1.340 \\
\hline $020 \ldots \ldots \ldots \ldots$ & 1.015 & 290 & 765 & 1.005 \\
\hline $021 \ldots \ldots \ldots$ & 1.290 & 530 & 805 & 1.150 \\
\hline $022 \ldots \ldots \ldots \ldots$ & 1.290 & 440 & 765 & 1.490 \\
\hline $100 \ldots \ldots \ldots \ldots$ & 955 & 180 & 580 & 1.335 \\
\hline $101 \ldots \ldots \ldots \ldots$ & 1.015 & 220 & 610 & 1.145 \\
\hline $102 \ldots \ldots \ldots \ldots$ & 1.250 & 190 & 720 & 1.740 \\
\hline $110 \ldots \ldots \ldots \ldots$ & 1.325 & 215 & 540 & 1.365 \\
\hline $111 \ldots \ldots \ldots \ldots$ & 1.250 & 410 & 780 & 1.415 \\
\hline $112 \ldots \ldots \ldots \ldots$ & 1.015 & 150 & 725 & 1.240 \\
\hline $120 \ldots \ldots \ldots \ldots$ & 975 & 470 & 720 & 1.235 \\
\hline $121 \ldots \ldots \ldots \ldots$ & 1.055 & 315 & 745 & 1.565 \\
\hline $122 \ldots \ldots \ldots \ldots$ & 1.290 & 420 & 720 & 1.155 \\
\hline $200 \ldots \ldots \ldots \ldots$ & 1.210 & 170 & 740 & 1.140 \\
\hline $201 \ldots \ldots \ldots \ldots$ & 1.115 & 145 & 610 & 1.400 \\
\hline $202 \ldots \ldots \ldots \ldots$ & 1.250 & 400 & 635 & 1.355 \\
\hline $210 \ldots \ldots \ldots \ldots$ & 1.330 & 440 & 780 & 1.310 \\
\hline $211 \ldots \ldots \ldots$ & 1.365 & 200 & 655 & 1.265 \\
\hline $212 \ldots \ldots \ldots \ldots$ & 1.675 & 505 & 690 & 1.420 \\
\hline $220 \ldots \ldots \ldots \ldots$ & 1.015 & 180 & 680 & 1.270 \\
\hline $221 \ldots \ldots \ldots \ldots$ & 1.365 & 165 & 845 & 1.385 \\
\hline $222 \ldots \ldots \ldots \ldots$ & 1.405 & 385 & 705 & 1.220 \\
\hline \multicolumn{5}{|l|}{ MEDIAS } \\
\hline $\mathbf{N}_{0} \ldots \ldots \ldots \ldots$ & 1.244 & 350 & 668 & 1.232 \\
\hline $\mathbf{N}_{1} \ldots \ldots \ldots \ldots$ & 1.126 & 286 & 682 & 1.355 \\
\hline $\mathbf{N}_{2} \ldots \ldots \cdots \cdots$ & 1.303 & 288 & 704 & 1.307 \\
\hline $\mathbf{P}_{0} \ldots \ldots \ldots \ldots$ & 1.170 & 223 & 626 & 1.328 \\
\hline $\mathbf{P}_{1} \ldots \ldots \ldots \ldots$ & 1.314 & 346 & 679 & 1.292 \\
\hline $\mathbf{P}_{2} \ldots \ldots \ldots \ldots$ & 1.189 & 355 & 750 & 1.275 \\
\hline $\mathbf{K}_{0} \ldots \ldots \ldots \ldots$ & 1.130 & 306 & 668 & 1.207 \\
\hline $\mathbf{K}_{1} \ldots \ldots \ldots \ldots$ & 1.224 & 292 & 719 & 1.316 \\
\hline $\mathbf{K}_{\mathrm{z}} \ldots \ldots \ldots \ldots$ & 1.318 & 326 & 688 & 1.372 \\
\hline
\end{tabular}


Vol. 41, Art. n.o 3

\subsubsection{Estação Experimental de Campinas}

No experimento realizado em Campinas, no ano agrícola 1968/69, para a produção de massa verde, o coeficiente de variação correspondeu a $9,9 \%$.

A adição de potássio apresentou efeito negativo, ao passo que o fósforo e o nitrogênio provocaram aumentos significativos.

Os efeitos linear e quadrático do fósforo foram significativos ao nível de 5 e $1 \%$ e os aumentos proporcionados para $\mathbf{P}_{1}$ e $\mathbf{P}_{2}$ foram respectivamente 14,9 e 4,8\%. Também significativo ao nível de $5 \%$, foi o efeito linear do nitrogênio, enquanto o efeito quadrático não foi significativo.

As interações dupla e tripla apresentaram efeito altamente significativo, somente apresentando efeito significativo a $5 \%$ para NP.

Para a produção de sementes, na análise estatística dos resultados, o coeficiente de variação foi $24,6 \%$.

A adição de nitrogênio, fósforo e potássio praticamente não modificou as produções.

Os efeitos linear e quadrático do fósforo, nitrogênio e potássio não apresentaram significância estatística, ocorrendo o mesmo com as interações duplas e triplas.

Apesar disso, a adição de $\mathbf{N}_{2}$ proporcionou aumentos de $4,7 \%$. O efeito do fósforo aumentou $12,3 \%$ para o $P_{1}$ e $1,6 \%$ para o $P_{2}$, e o potássio causou aumentos de 8,3 e $16,6 \%$ respectivamente para o $K_{1}$ e $\mathrm{K}_{2}$.

\subsubsection{Estação Experimental de Tietê}

No experimento realizado em Tietê, no ano agrícola 1968/69, para a produção de massa verde, o coeficiente de variação foi $16,6 \%$.

A adição de nitrogênio apresentou leve decréscimo na produção, não apresentando significância estatística, ocorrendo o mesmo com seus efeitos linear e quadrático.

O efeito do fósforo foi altamente significativo, apresentando aumento de produção de $12,1 \%$ para o $P_{1}$ e $25,3 \%$ para $\circ \mathrm{P}_{2}$, com significância a $5 \%$ para o componente linear.

O efeito da adição de potássio não foi significativo, tanto para o componente linear como quadrático, apresentando aumento de $8,3 \%$ apenas para $\circ \mathbf{K}_{2}$.

A interação dupla NP apresen. tou efeito significativo ao nível de $5 \%$, ao passo que NK e a interaçâo tripla NPK foram altamente significativas ao nível de $1 \%$.

$\mathrm{Na}$ análise estatística dos resultados, para a produção de sementes, o coeficiente de variação foi $17,9 \%$.

A adição de nitrogênio causou efeito depressivo na produção de sementes altamente significativo; para o efeito linear, porém, a significância foi negativa ao nível de $1 \%$ e para o efeito quadrático foi positiva ao nível de $5 \%$. O efeito do fósforo foi altamente significativo, provocando aumentos de $55,1 \%$ para o $P_{1}$ e $59,2 \%$ para o $\mathrm{P}_{2}$. Os efeitos linear e quadrático foram igualmente altamente significativos. 
A aplicação do potássio não apresentou efeito significativo, ocorrendo o mesmo com seus componentes linear e quadrático.

As interações duplas NP e NK apresentaram efeito altamente significativo, ao passo que PK foi significativo apenas ao nível de $5 \%$.

A interação NPK apresentou efeito altamente significativo.

\subsubsection{Estação Experimental de Tatuí}

No experimento realizado durante o ano agrícola 1968/69, na Estação Experimental de Tatuí para a produção de massa verde, o coeficiente de variação correspondeu a $15,9 \%$.

A adição de nitrogênio causou pequeno aumento na produção, sem apresentar efeito significativo, ocorrendo o mesmo com seus componentes linear e quadrático. Os aumentos para $\mathrm{N}_{1}$ e $\mathrm{N}_{2}$ foram respectivamente 5,3 e $9,8 \%$.

$O$ efeito da adição de fósforo foi altamente significativo, proporcionando aumentos de 10,0 e $28,4 \%$ respectivamente para $\mathbf{P}_{1}$ e $\mathbf{P}_{2}$. $\mathrm{O}$ componente linear apresentou efeito altamente significativo, enquanto o quadrático apresentou efeito não-significativo.

A adição de potássio não apresentou significância estatística, ocorrendo o mesmo com seus componentes linear e quadrático. Houve aumento de $10,9 \%$ para $K_{1}$ e $6,5 \%$ para $\mathrm{K}_{2}$.

As interações duplas não alcançaram significância, enquanto $a$ inte- ração tripla alcançou significância, mas ao nível de $5 \%$.

Para a produção de sementes, a análise estatística dos resultados apresentou um coeficiente de variarespectivamente 8,5 e $19,8 \%$.

A adição de nitrogênio não apresentou efeito significativo, ocorrendo o mesmo com seus componentes linear e quadrático. Houve aumento na produção de $2,1 \%$ para o $\mathrm{N}_{1}$ e $5,4 \%$ para o $\mathrm{N}_{2}$.

$O$ efeito da adição de fósforo foi significativo ao nível de $5 \%$; seu componente linear apresentou efeito altamente significativo, enquanto o componente quadrático não apresentou significância estatística. Os aumentos proporcionados por $\mathbf{P}_{1}$ e $\mathbf{P}_{2}$ foram respectivamente 8,5 e $19,8 \%$.

$\Theta$ emprego do potássio não apresentou significância estatística, havendo somente aumento de 7,6\% para o $\mathrm{K}_{\mathbf{1}}$.

As interações duplas não alcançaram significância, ocorrendo o mesmo com a interação tripla.

\subsubsection{Estação Experimental de Ribeirão Preto}

Realizado no ano agrícola de 1968/69 na Estação Experimental de Ribeirão Preto, o presente experimento apresentou para a produção de massa verde coeficiente de variação de $15,3 \%$.

O emprego de nitrogênio, fósforo e potássio não apresentou efeito significativo, ocorrendo o mesmo com seus componentes lineares e quadráticos. 
Vol. 41, Art. n. ${ }^{\circ} 3$

O nitrogênio proporcionou aumentos de 7,6 e $7,2 \%$ respectivamente para $\mathrm{N}_{1}$ e $\mathrm{N}_{2}$.

O fósforo apresentou leve decréscimo na produção de massa verde.

O potássio, de $4,7 \%$ para o $\mathrm{K}_{1}$ e $8,2 \%$ para o $\mathrm{K}_{2}$.

As interações duplas NP e NK não alcançaram significância; PK e NPK apresentaram efeito altamente significativo.

Um coeficiente de variação de $22,2 \%$ foi apresentado pela análise estatística dos resultados de produção de sementes.

Os efeitos da adubação de nitrogênio, fósforo e potássio não alcançaram significância, como os de seus componentes lineares e quadráticos.

Os aumentos proporcionados pelo emprego do nitrogênio foram 10,0 e $6,1 \%$ respectivamente para $\mathrm{N}_{1}$ e $\mathrm{N}_{2}$.

O P apresentou leve decréscimo na produção de sementes.

$\mathrm{K}_{1}$ e $\mathrm{K}_{2}$ causaram aumentos respectivamente de 9,0 e $13,7 \%$, não apresentando significância.

As interações duplas e triplas não alcançaram significância.

\section{CONCLUSÓES}

Analisando os dados relatados, podemos concluir que nas diferentes experiências realizadas, a crotalária reagiu diferentemente aos fertilizantes empregados, tanto quanto à produção de massa verde como de sementes, para as diferentes localizações.
Em Campinas, o efeito do fósforo foi altamente significativo nos dois anos agrícolas estudados, ao passo que o nitrogênio apresentou significância para 1968 , para a produção de massa verde.

Na mesma região, para a produção de sementes, fósforo e potássio apresentaram significância para o experimento no ano agrícola 1967.

Na região de Tietê, o nitrogênio foi significativo para 1967 e o fósforo no ano seguinte. O potássio não apresentou significância para a produção de massa verde.

Para a produção de sementes, houve significância no primeiro ano para o nitrogênio e no seguinte para nitrogênio e fósforo.

Na região de Tatuí, apenas o fósforo foi significativo para a produção de massa verde, nos dois anos agrícolas, apresentando significância para o segundo ano para a produção de sementes.

Na região de Ribeirão Preto, não houve significância nos dois anos agrícolas para qualquer tratamento, para a produção de massa verde, porém a interação NPK foi significativa em ambos.

Para a produção de sementes, apenas a interação NPK apresentou significância, mesmo assim somente para o primeiro ano de cultivo.

Podemos concluir ser aconselhável uma adubação fosfatada na maioria dos solos do Estado de São Paulo, assim como uma adubação completa naqueles mais pobres, para que haja aumento na produção de massa verde e sementes. 
INFLUENCE OF NPK FERTIIIZATION IN SUNNHEMP CROP

\section{SUMMARY}

In this paper the influence of NPK fertilization in the production of sunnhemp (Crotalaria juncea L.) regarding fibers and seeds was studied. The experiments were conducted during two years in four different reglons of Săo Paulo State.

The data showed that the best results were obtained with the application of phosphorus.

\section{REFERENCLAS BIBLIOGRAFICAS}

1. BRAsh. Serviço Nacional de Pesquisas Agronômicas. Comissåo de Solos. Levantamento de reconhecimento dos solos do Estado de Săo Paulo. Rio de Janeiro, Ministério da Agricultura, 1960. 634p.

2. Lovadint, L. A. C.; SALGADO, A. L. B.; MTYASAKA, S. Efeito da época de plantio e da poda na produçäo de massa verde e sementes de Crotalaria juncea L. Bragantia, Campinas, 29:25-30, 1970.

3. MEDINA, J. C.; CIARAMELLO, D.; PETTINELII, A. Resultados experimentais com a cultura da Crotalaria juncea $L$. como planta produtora de celulose para papel. Bragantia, Campinas, 20:659-668, 1961.

4. SAlGado, A. L. B. Plantas fibrosas no Estado de São Paulo. In: SEMINARIO DE FITOTECNIA, Piracicsba, ESALQ-USP, 1973. p.64-90. 